\title{
UTILISATION DU PHYTOPLANCTON POUR ESTIMER LA PRODUCTION PISCICOLE POTENTIELLE DES ÉTANGS.
}

\author{
J. BARBE (1), O. SCHLUMBERGER (2) et N. BOURETZ (3)
}

(1) CEMAGREF, Unité de Recherches Biologie des Ecosystèmes Aquatiques. 3 bis Quai Chauveau, CP 220, 69336 Lyon Cedex 09, France.

(2) CEMAGREF, Unité de Recherches Ressources Ichtyologiques en Plans d'Eau. Domaine de Lavalette, BP 5095, 34033 Montpellier Cedex 01, France.

(3) 7 impasse du Pas du Loup, 30700 Uzès, France.

\section{RÉSUMÉ}

Pour faciliter la gestion des étangs de pisciculture, il est nécessaire d'avoir un moyen pour estimer en cours de saison si l'objectif de production sera atteint, et éventuellement, intervenir sur la qualité du milieu. Les méthodes d'estimation et de prévision mentionnées dans la littérature ne sont pas applicables aux étangs de pisciculture, milieux fortement anthropisés. A partir de données (physico-chimie, plancton, production piscicole) collectées sur 36 étangs en France, les auteurs proposent une méthode de prévision de la récolte piscicole à partir du phytoplancton (groupe prédominant et teneur en chlorophylle a). A l'usage, cette méthode s'est avérée satisfaisante pour prévoir des productions nettes jusqu'à plus de $500 \mathrm{~kg} / \mathrm{hectare}$. piscicole.

Mots-clés : étang de pisciculture, phytoplancton, chlorophylle $a$, production

\section{UTILIZATION OF PHYTOPLANKTON TO ESTIMATE THE POTENTIAL FISH PRODUCTION IN PONDS.}

\begin{abstract}
Informations about the potential production level of a fertilized fishpond are indispensable for its rational management. For fisheries, various methods are existing to correlate fish-biomass and -yield of lakes to their morphometry, physico-chemical and biological parameters. Such methods proved to be unappropriate for the fertilized fish-ponds studied by the authors in France ( 36 different locations). This can be explained by the pecularities of such water bodies : strong anthropication, low diversity of the stocked (cultivated) fish species, optimized fertilization.
\end{abstract}

From the data collected by the authors, statistical treatment evidenced links between phytoplankton types, chlorophyll densities and fish production levels. Combining both five typical groups of phytoplankton and five different concentrations of chlorophyll a arranged into a grid, it is possible to determine the potential fish production of the ponds (up to over $500 \mathrm{~kg} / \mathrm{ha}$ ).

Key-words : fish-pond, phytoplankton, chlorophyll a, fish production. 


\section{INTRODUCTION}

Sauf cas d'alimentation directe du poisson avec une nourriture exogène, la production piscicole d'un plan d'eau dépend de la richesse du milieu (plancton essentiellement) qui est elle-même fonction des apports de fertilisants minéraux et/ou organiques. La connaissance de la capacité de production d'un étang de pisciculture est indispensable pour pouvoir le gérer et estimer en cours de saison si l'objectif de production sera atteint compte tenu de la fertilisation pratiquée.

Cette production potentielle est la production que devrait avoir un étang empoissonné de manière optimale en quantité et qualité, compte tenu de sa morphologie générale.

\section{SITUATION DU PROBLÈME}

\section{Problème à résoudre}

Chargés d'apporter un appui technique pour la gestion d'étangs de pisciculture, les auteurs ont cherché à disposer d'indicateur(s) de la production potentielle d'un étang de pisciculture à partir de mesures faites en cours de saison. L'objectif étant de prévoir la quantité de poisson que l'on récoltera à la vidange.

L'étude a été réalisée sur des étangs de pisciculture, avec élevage polyspécifique, apports de fertilisants organiques ou minéraux mais sans nourrissage. Trente-six sites différents (Tableau I) ont été suivis, dont 10 plusieurs années de suite ; au total, 49 séries de données sont disponibles. Ces plans d'eau sont situés dans différentes régions de France (Lorraine, Brenne, Forez et Dombes), et leur surface est comprise entre 0,6 et 170 hectares, 6 sites ayant une superficie dépassant 20 hectares. La récolte (production nette) varie de 60 à $628 \mathrm{~kg} / \mathrm{ha} /$ an suivant les sites (moyenne : $232 \mathrm{~kg} /$ hectare).

Les paramètres suivants (CEMAGREF, 1986 à 1991) ont été mesurés pendant la période de croissance du poisson (d'avril à septembre) :

- physico-chimie : température, oxygène dissous en surface et au fond, transparence au disque de Secchi, conductivité, teneurs en $\mathrm{Ca}^{++}, \mathrm{Mg}^{++}, \mathrm{HCO}_{3}^{-}, \mathrm{CO}_{3}^{--}$, $\mathrm{NH}_{4}^{+}, \mathrm{NO}_{2}^{-}, \mathrm{NO}_{3}^{-}, \mathrm{N}$ minéral total, $\mathrm{N}$ total, $\mathrm{PO}_{4}^{---}$et $\mathrm{P}$ total ;

- phytoplancton : groupe dominant établi après dénombrement (les colonies sont comptabilisées comme un individu) et chlorophylle $a$;

- zooplancton : nombre d'individus/litre, biovolume décanté $(\mathrm{ml} / 100$ litres d'eau brute; organismes frais) et poids sec (passage à l'étuve : 48 heures à $120^{\circ} \mathrm{C}$ ) :

- production piscicole de chaque étang, relevée en hiver, au moment de la pêche annuelle par vidange de ces étangs.

Les relevés concernant la physico-chimie et le plancton ont été effectués au cours de 8 à 12 campagnes annuelles. Les contrôles débutent dès la fin du remplissage des étangs (habituellement en avril) et se terminent fin septembre. Mesures et prélèvements ont été faits sur toute la colonne d'eau, en 6 à 10 points suivant la superficie des sites.

Quelques remarques peuvent être faites concernant les étangs notés * dans le 


\section{Tableau I}

Base de données utilisée et application de la méthode proposée. Détermination de la production piscicole nette potentielle d'un étang à partir du (des) groupe(s) de phytoplancton prédominant(s) au cours de la saison, et des concentrations moyennes en chlorophylle $a$.

\section{Table I}

Determination of the potential net fish production of a pond from the dominant populations of phytoplankton and mean chlorophyll a concentrations.

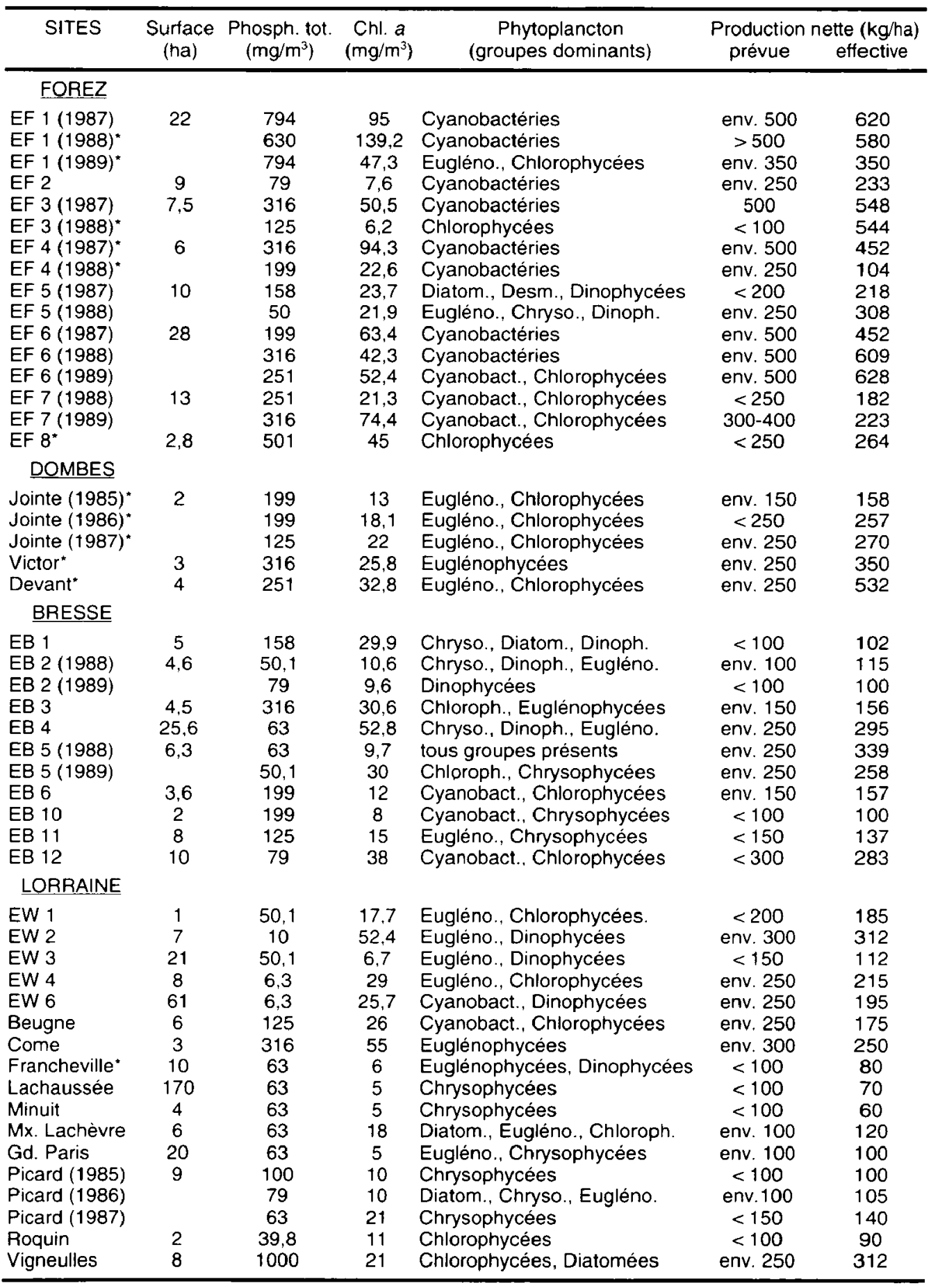


- EF1 (saisons 1988 et 1989) : pas de mortalités de poissons malgré la présence de Cyanobactéries en 1988 ; installation d'un aérateur de surface pendant l'été 1989 ;

- EF3 (saison 1988) : forte augmentation de la densité de zooplancton de grande taille par rapport à la saison précédente (apports probables de matières organiques) ;

- EF4 (saisons 1987 et 1988) : mortalités sur une partie de la production en 1987 (carpes de 2 étés) ; faible production en 1988 due à l'absence de reproduction des carpes ;

- EF8 : des apports de sulfate de cuivre $\left(30-50 \mathrm{mg} \mathrm{Cu} / \mathrm{m}^{3}\right)$ ont été effectués pour éviter le développement de Cyanobactéries;

- Etangs Jointe, Victor et Devant : la fertilisation organique à base de lisier et fiente de volailles a favorisé le développement d'Euglénophycées.

\section{Les indicateurs disponibles}

Les paramètres physico-chimiques et biologiques du milieu qui peuvent être utilisés se situent dans les différents compartiments de la chaîne trophique : éléments dissous, phytoplancton et zooplancton.

\section{physico-chimie}

En pisciculture d'étang, les gammes de concentrations considérées comme optimales pour les principaux sels minéraux dissous sont les suivantes (BILLARD et MARIE, 1980 ; WURTZ-ARLET, 1980 ; BOYD, 1982) :

- calcium $\mathrm{Ca}^{++}=$plus de $8,5 \mathrm{mg} \mathrm{Ca}{ }^{++} /$itre, soit $30 \mathrm{mg} \mathrm{CaCO} /$ litre. Le calcium est un facteur limitant de la production aquatique en général ; les eaux les plus productives en contiennent plus de $50 \mathrm{mg} / \mathrm{litre}$;

- azote inorganique dissous $\left(\mathrm{N}-\mathrm{NH}_{4}+\mathrm{N}-\mathrm{NO}_{3}\right)=0,8$ à $4 \mathrm{mg} / \mathrm{litre}$;

- orthophosphates $\mathrm{PO}_{4}=0,2$ à $0,5 \mathrm{mg} \mathrm{PO} /$ /itre ;

- rapport $\mathrm{PO}_{4} /\left(\mathrm{N}_{\mathrm{N}} \mathrm{NH}_{4}+\mathrm{N} . \mathrm{NO}_{3}\right)=1 / 4$ à $1 / 10$. Si la proportion est supérieure à $1 / 4$, on observe fréquemment un développement de Cyanobactéries; des valeurs de $1 / 8$ à 1/10 favorisent les Chlorophycées.

L'azote dissous (formes $\mathrm{N}^{\mathrm{NH}} \mathrm{NH}_{4}$ et $\mathrm{N}_{\mathrm{NO}}$ ) et les orthophosphates $\left(\mathrm{PO}_{4}\right)$ sont utilisés par le phytoplancton pour son développement.

En pratique, les observations de terrain montrent que dans de nombreux étangs, les flux de consommation d'azote et de phosphore sont tels que leurs concentrations instantanées respectives sont inférieures à l'optimum théorique d'un facteur 10 voire plus, alors que le plancton est abondant (Secchi : $60-80 \mathrm{~cm}$ ), avec une production piscicole nette de 300 à $500 \mathrm{~kg} /$ hectare.

\section{Transparence de l'eau mesurée au disque de Secchi}

Ce critère est à la base de la gestion des étangs de pisciculture dans toute l'Asie du Sud-Est, où les espèces produites sont essentiellement filtreuses et planctonophages. II permet d'apprécier la densité des particules (matières en suspension, et surtout plancton) 
présentes dans l'eau. Lorsque la transparence se maintient entre 30 et $60 \mathrm{~cm}$, on considère que la densité de plancton est correcte pour assurer une bonne production (BILLARD et MARIE, 1980 ; WURTZ-ARLET, 1980 ; BOYD, 1982). Cette mesure est perturbée si le sédiment est remis en suspension (action du vent sur la masse d'eau, activité des poissons sur le fond) ou lorsque l'eau est fortement colorée par des substances humiques (environnement forestier).

En pratique : dans certains étangs, la transparence de l'eau peut rester importante pendant toute la période de croissance des poissons, bien que la production finale soit correcte. Dans le cas d'un plan d'eau entouré d'une importante ceinture de macrophytes, les valeurs suivantes ont été relevées (moyennes saisonnières) : azote $\left(\mathrm{N} \mathrm{NH}_{4}+\mathrm{N} . \mathrm{NO}_{3}\right)$ et orthophosphates dissous : moins de $0,05 \mathrm{mg} / \mathrm{litre}$ pendant la saison pour chacun de ces deux éléments fertilisants ; transparence au disque de Secchi élevée : stable, à environ $2 \mathrm{~m}$. La production nette a atteint $225 \mathrm{~kg}$ de poisson/hectare.

Un tel résultat s'explique par le développement de bactérioplancton favorisé par un rapport C/N élevé (BERARD, 1993 ; AVNIMELECH et al., 1994), grâce à la présence de déchets végétaux abondants (source de carbone). La biosynthèse de protéines microbiennes explique les faibles teneurs en azote dissous mesurées. Le bactérioplancton est consommé directement par du zooplancton de petite taille (Ciliés, Rotifères, Cladocères) dont l'influence sur la transparence est réduite.

\section{Plancton}

Le phytoplancton est utilisé classiquement comme indicateur du degré d'eutrophisation des plans d'eau douce (WURTZ, 1958 ; BARBE et al., 1990). Son développement dépend de la richesse du milieu en azote et phosphore, et de la prédation exercée par le zooplancton. Mais le développement d'une forte biomasse algale n'est pas suffisant pour pouvoir obtenir un rendement piscicole élevé. La nature des espèces d'algues, les dimensions des cellules et leur structure doivent permettre leur bonne intégration dans la chaîne alimentaire aboutissant au poisson (BALVAY, 1995 ; LAZZARO et LACROIX, 1995). En outre, un développement algal excessif peut entraîner l'apparition de phases de désoxygénation nocturne (respiration de la biomasse algale); des mortalités massives sont à craindre également, avec toutes les conséquences biologiques et physico-chimiques que cela induit.

Le zooplancton est consommé directement par les jeunes stades des différentes espèces de poisson, et par les adultes non carnivores (Cyprinidés). Sa répartition dans l'étang est généralement plus hétérogène que celle du phytoplancton. Les alevins consomment initialement surtout des Rotifères et des stades naupliens de Copépodes ; les poissons plus âgés (à partir de 3-4 semaines) exercent leur prédation sur les formes adultes de Copépodes et les Cladocères. Pour utiliser le plancton comme indicateur de la production potentielle d'un étang, il faut pouvoir estimer si la chaîne trophique phytoplancton - zooplancton - poisson est bien développée qualitativement et quantitativement. La densité du zooplancton (nombre d'individus/litre) ou le biovolume décanté ( $\mathrm{ml}$ zooplancton/100 litres) sont des indicateurs de production importants, mais les tailles relatives et les régimes alimentaires des différents groupes planctoniques présents, ainsi que la prédation exercée par les poissons (Cyprinidés, quel que soit leur âge, et juvéniles des autres groupes) doivent être pris en compte. 
Le zooplancton a été utilisé en Pologne par GRYGIEREK (1979) comme aide à la gestion pour les étangs fertilisés destinés au grossissement de carpes (C. carpio) en monoculture pendant leur première, seconde ou troisième année. En tenant compte:

- de la taille des organismes zooplanctoniques présents ;

- des proportions relatives entre les différents groupes;

- des rendements obtenus en fin d'année lors des pêches.

La méthode proposée permet de définir des densités optimales de carpes pour ce mode d'élevage particulier. Les meilleures productions (300-330 kg net/hectare) sont obtenues quand les Daphnies (Daphnia sp.) constituent 20 à $30 \%$ du zooplancton en juillet et août, période de croissance maximale pour les carpes. Malheureusement, cette méthode ne s'applique que pour le grossissement de la carpe en monoculture, ce qui ne permet pas d'extrapoler cette méthode aux étangs que nous étudions, avec polyculture et reproduction sur place de certaines espèces.

\section{LES DIFFÉRENTES APPROCHES}

\section{Les méthodes existantes}

Dans les grands plans d'eau dulçaquicoles exploités par pêche aux engins, différentes méthodes ont été proposées pour estimer soit la production piscicole (" fish yield ", en kg/unité de surface), soit la biomasse de poissons présente ("fish standing crop ", cheptel existant sur le secteur) à partir : 1956) ;

- des substances dissoutes totales (RAWSON, 1951 ; NORTHCOTE et LARKIN,

- de la profondeur moyenne (RAWSON, 1952);

- de l'indice morphoédaphique (RYDER et al., 1974) et des formules dérivées (revue dans OGLESBY, 1982) ;

- de la biomasse de phytoplancton (OGLESBY, 1977a et b ; JONES et HOYER, 1982 ; BIRO et VOROS, 1990); 1982).

- de la biomasse de macrobenthos (MATUSZEK, 1978 ; HANSON et LEGGETT,

Une revue de ces méthodes a été faite par LEACH et al. (1987).

MOREAU et DE SILVA (1991) montrent que sur des lacs et réservoirs d'Asie, suivant les sites, c'est l'une ou l'autre de ces méthodes qui fournit les meilleures estimations des rendements de la pêche.

Pour les plans d'eau de petite surface non anthropisés, la formule de LEGER-HUET (HUET, $1949 ; 1964 ; 1970$ ) est fréquemment utilisée. HANSON et LEGGETT (1982) ont proposé, à partir d'une compilation de relevés sur 26 plans d'eau de l'hémisphère Nord, une méthode de détermination de la biomasse de poissons en fonction de la concentration moyenne en phosphore total dissous dans le milieu. Pour leur part, DOWNING et al. (1990) ont repris des données bibliographiques concernant la production biologique de lacs de différentes zones géographiques et de divers niveaux trophiques. Ils montrent que 
la production biologique des communautés de poissons concernées est relativement mieux corrélée avec la production primaire (en $\mathrm{g}$ de Carbone $/ \mathrm{cm}^{2} / \mathrm{an}$ ) qu'avec la concentration en phosphore total ou l'indice morphoédaphique.

\section{Limites de ces méthodes dans le cas des étangs piscicoles}

Par rapport à d'autres plans d'eau, les étangs de pisciculture constituent des milieux particuliers du fait surtout de leurs modes de gestion et d'exploitation :

- le stock de poissons est récupéré en totalité lors de la vidange, et est égal, dans ces conditions, à la biomasse. En conséquence, les méthodes mises au point pour estimer les productions ou rendements par pêche aux engins ne s'appliquent pas sur de tels sites. Dans les étangs, ce stock de poissons est optimisé (association d'espèces, gamme de tailles et d'âges) en fonction de la morphologie et des potentialités du site, et de la durée du cycle de production ( 1 an le plus souvent). Le peuplement de poisson est peu variable d'un étang piscicole à l'autre : carpe (Cyprinus carpio), tanche (Tinca tinca), gardon (Rutilus rutilus) et rotengle (Scardinius erythrophtalmus) avec brochet (Esox lucius), plus rarement sandre (Stizostedion lucioperca) ou perche (Perca fluviatilis), comme carnivores d'accompagnement ;

- leur forte anthropisation (vidange tous les 1 ou 2 ans, entretien des bords, assec, éventuellement chaulage) limite le développement des macro-organismes aquatiques végétaux et animaux (benthos), et ne permet pas d'appliquer la formule de LEGER-HUET, dans laquelle la production piscicole est fonction directe de la densité d'herbiers ;

- leurs dimensions sont restreintes : la gamme des profondeurs maximales va de 1,5 à 5 mètres, et la surface moyenne des étangs de pisciculture en France est de 3-4 hectares ;

- l'optimisation des apports de fertilisants (à base de $N$ et $P$ ), à la fois en quantités et dans le temps, permet leur utilisation rapide par la chaîne trophique. L'intensité du flux de matière et d'énergie qui y circule est à l'origine des faibles teneurs instantanées mesurées pour l'azote et le phosphore dissous, inférieures aux concentrations optimales conseillées (BARBE et al., 1991).

Ces particularités ont pour conséquence que les formules basées sur des teneurs en éléments dissous comme la formule de l'indice morpho-édaphique de RYDER et al. (1974) utilisant les solides totaux dissous (TDS : total dissolved solids, résidu sec obtenu après évaporation), ou la formule proposée par HANSON et LEGGETT (1982) basée sur le phosphore total, ne peuvent pas s'appliquer ici. En effet, dans le cas de l'indice morpho-édaphique, les teneurs en solides totaux dissous peuvent être extrêmement variables d'un site à l'autre, mais aussi pour un même site, selon l'agitation de l'eau, l'activité des poissons sur le fond, la présence de macrophytes, les apports d'engrais et d'amendements effectués, etc. D'autre part, seule une fraction des phosphates totaux mesurés est assimilable par le phytoplancton.

\section{Essai d'application aux étangs}

L'application de la méthode de HANSON et LEGGETT (1982) aux 49 séries de données recueillies sur les étangs de pisciculture, montre que la corrélation entre la production piscicole ( $\mathrm{kg} / \mathrm{hectare}$ ) et la concentration moyenne annuelle en phosphore total dissous ( $\mu$ g/litre) est faible $(R=0,48)$. Pour une même concentration en $P$ total dissous, on peut obtenir des productions qui peuvent différer d'un facteur 8 à 10 suivant les sites. 
De la même manière, l'application de la méthode de calcul d'après OGLESBY (1977a) (Figure 1) indique une corrélation peu élevée $(R=0,61)$ entre la production piscicole ( $\mathrm{kg} / \mathrm{hectare}$ ) et la concentration en chlorophylle a (maximum de printemps, avril-mai) exprimée en $\mathrm{mg} / \mathrm{m}^{3}$. A partir d'une même concentration en chlorophylle $a$, les variations de production peuvent être très fortes d'un étang à l'autre, bien que plus faibles qu'à partir du phosphore total.

En conclusion, une relation statistique établie par régression à partir de ces différents facteurs n'est pas utilisable pour déterminer à l'avance une production piscicole.

relation production nette / chlorophylle a

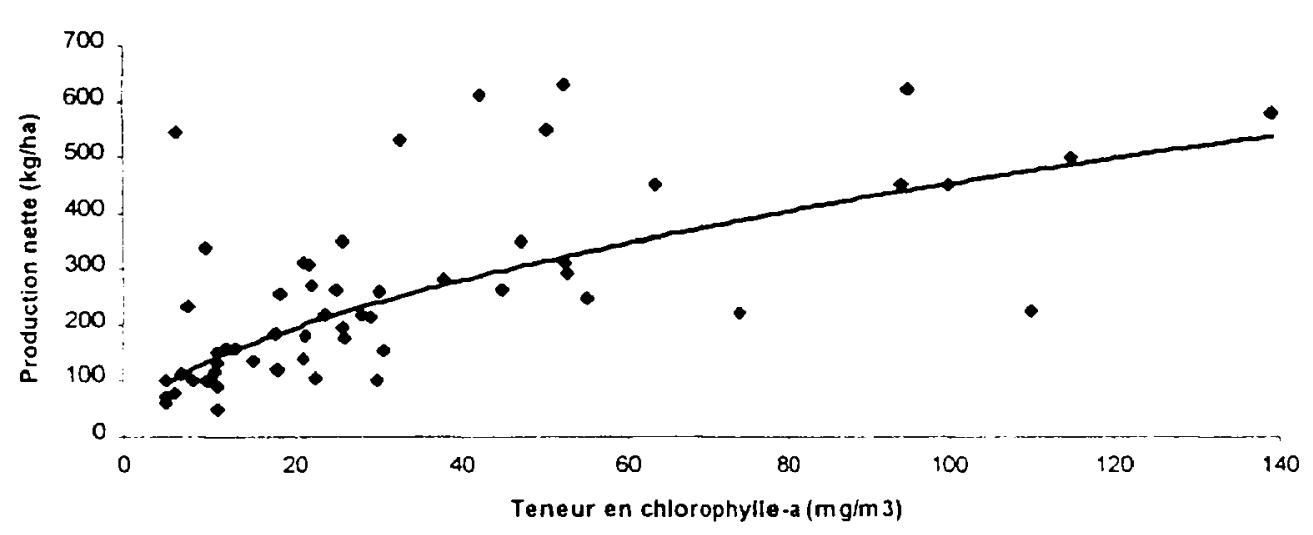

Figure 1

Formule d'OGLESBY (1977a) appliquée à la production piscicole nette annuelle et la concentration en chlorophylle a dans les étangs étudiés (CEMAGREF, 1986 à 1991).

\section{Figure 1}

OGLESBY (1977a) plot of annual net fish yield on chlorophyll a concentration in the studied fishponds (CEMAGREF, 1986 to 1991).

\section{Méthode proposée}

Compte tenu de ces contraintes, la méthode proposée ici est basée sur le phytoplancton, en combinant des données qualitatives et des données quantitatives : d'une part les peuplements phytoplanctoniques présents, et d'autre part, la concentration en chlorophylle a donnant une indication sur la densité de ces peuplements. Nous utiliserons par la suite la concentration moyenne estivale en chlorophylle a (période avril-septembre).

Cinq groupes de phytoplancton selon l'analyse de WURTZ (1958), ont été retenus. Ces associations phytoplanctoniques sont considérées comme représentatives à la fois de niveaux croissants de minéralisation et d'eutrophisation (effet "bottom-up ") et de la prédation qu'elles subissent de la part du zooplancton brouteur-filtreur (effet " top-down ").

Ces groupes d'algues sont identiques à ceux utilisés pour la méthode de diagnose rapide des plans d'eau (" indice trophique planctonique ", BARBE et al., 1990) : 
- groupe I: Desmidiées et Diatomées, dans les étangs oligotrophes dont l'eau est très peu minéralisée, et plus ou moins acide ;

- groupe II : Dinophycées (Péridiniens) et Chrysophycées, si l'étang a un peu de vase mais reste pauvre en matières minérales dissoutes, avec présence de matières organiques en suspension ;

- groupe III : Chlorococcales et Volvocales, lorsqu'une fertilisation minérale prédomine par rapport aux apports organiques ; ces plans d'eau eutrophes ont régulièrement des teneurs faibles en oxygène dissous en profondeur pendant la saison chaude ;

- groupe IV : lorsque la fertilisation organique est intense (fortes teneurs en MES organiques), le peuplement dominant est constitué d'Euglénophycées, hétérotrophes facultatifs capables de consommer des particules de matière organique en suspension ;

- groupe $V$ : si la fertilisation minérale est déséquilibrée (azote devenant facteur limitant par rapport au phosphore, rapport $P O / N>1 / 4)$, il y a prédominance de Cyanobactéries capables d'utiliser l'azote sous forme gazeuse dissoute dans l'eau. Ces proliférations s'observent également en cas de relargage (désorption) de phosphore par le sédiment, consécutif à une sous-saturation en oxygène près du fond (teneurs inférieures à $1,5 \mathrm{mg} \mathrm{O} /$ itre).

D'une manière générale, un développement de Cyanobactéries, même s'il permet souvent une production élevée, représente une situation de déséquilibre où les toxines et l'anoxie constituent un risque potentiel permanent de mortalité pour le stock de poissons.

Parallèlement à ces différents groupes de phytoplancton, et à partir des mesures effectuées sur le terrain (prélèvements dans la colonne d'eau sur 6 à 10 points), 4 classes de valeurs ont été retenues pour les concentrations moyennes estivales en chlorophylle $a$. Ces 4 classes se situent dans les catégories des plans d'eau oligotrophes à eutrophes définies par l'OCDE (1982) en raison de la vocation productive qu'ont par nature ces étangs :

- moins de $20 \mu \mathrm{g} / \mathrm{litre}$;

- de 20 à $50 \mu \mathrm{g} /$ litre ;

- de 50 à $100 \mu \mathrm{g} /$ litre ;

- plus de $100 \mu \mathrm{g} /$ litre.

A partir des groupes de phytoplancton et des classes de concentration en chlorophylle a (base de données présentée au Tableau I), les données ont été codées par classes pour une analyse en composantes multiples (ACM). La Figure 2 représente le plan 1-2 de l'ACM. Le pourcentage d'information restitué par l'analyse est de $50 \%$. L'axe horizontal F1 permet de séparer les étangs suivant le groupe phytoplanctonique, la quantité de chlorophylle a et la production piscicole nette. 


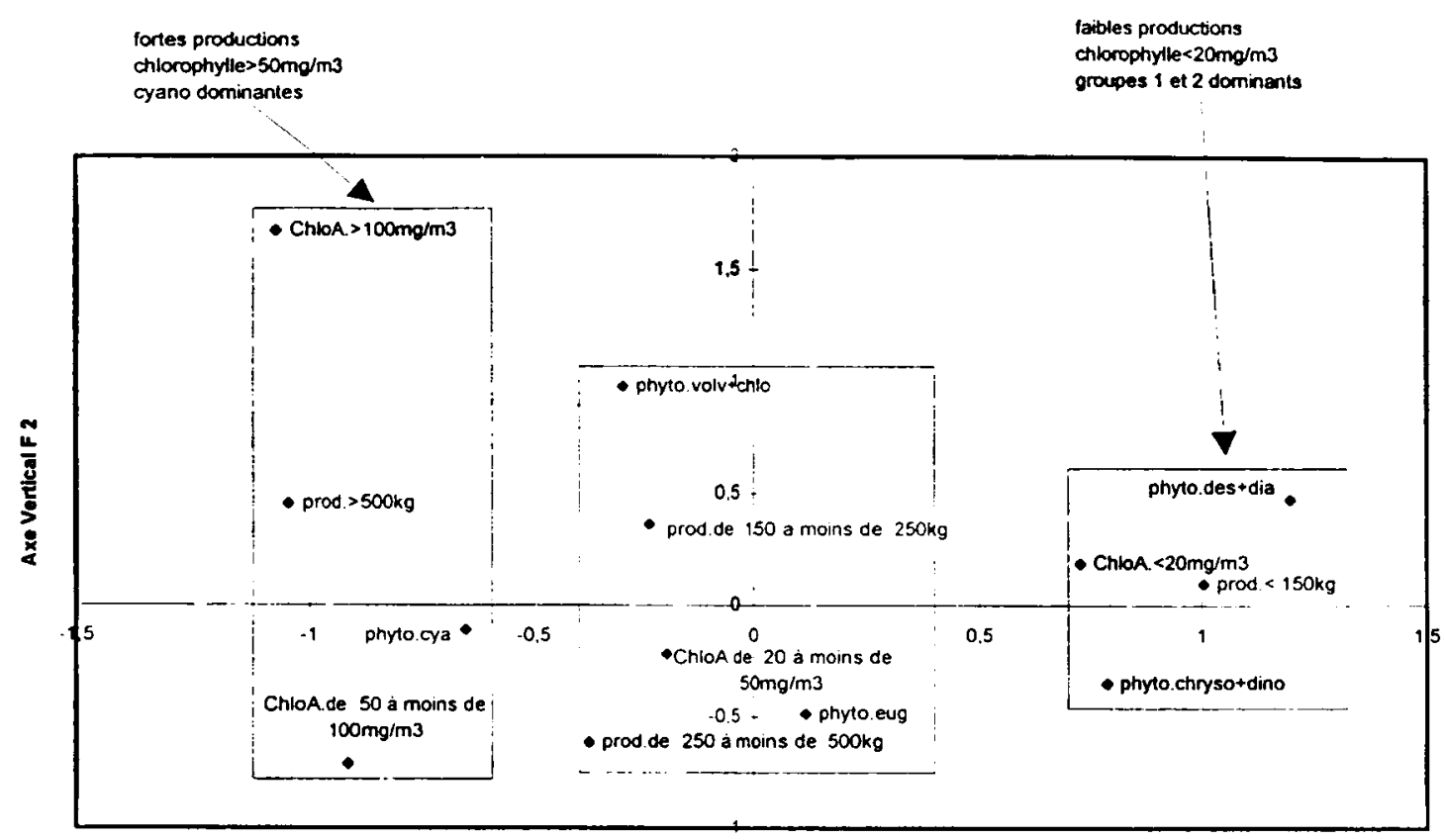

Axe Horizontal F 1

\section{Figure 2}

Axes 1 et 2 de l'ACM présentant les relations entre les teneurs en chlorophylle a ( $\mathrm{mg} /$ /litre) et la production piscicole nette ( $\mathrm{kg} / \mathrm{hectare})$ des étangs.

\section{Figure 2}

Relations between chlorophyll a concentration and fish net production in ponds (axes 1 and 2 of MCA).

Les groupes I (Diatomées + Desmidiées) et II (Chrysophycées + Dinophycées) du phytoplancton ont des contributions relatives (CTR) respectivement de 28 et $32 \%$. Ils sont corrélés à la fois aux faibles teneurs en chlorophylle $a$ et aux productions piscicoles inférieures à $150 \mathrm{~kg} /$ hectare (CTR : $86 \%$ ). En position opposée, le groupe $V$ (Cyanobactéries) est lié aux productions piscicoles supérieures à $500 \mathrm{~kg} / \mathrm{hectare}$, et aux teneurs en chlorophylle a dépassant $50 \mathrm{mg} / \mathrm{m}^{3}$. Les groupes III (Volvocales et Chlorococcales) et IV (Euglénophycées) sont en situation intermédiaire sur les axes, avec des productions piscicoles moyennes à faibles. L'étude des axes 2 et 3 permet de préciser leurs relations (Figure 3 : axes 1-3 de l'ACM).

Sur l'axe 3, les Cyanobactéries (groupe IV) sont corrélées avec les teneurs en chlorophylle comprises entre 50 et $100 \mathrm{mg} / \mathrm{m}^{3}$. Le groupe III (Volvocales et Chlorococcales) est corrélé avec des teneurs en chlorophylle comprises entre 20 et $50 \mathrm{mg} / \mathrm{m}^{3}$ et des productions piscicoles nettes de 250 à $500 \mathrm{~kg} /$ hectare. Sur l'axe 2, ce même groupe est opposé aux productions comprises entre 250 et $500 \mathrm{~kg} / \mathrm{hectare}$.

Dans le plan 2-3, les groupes de phytoplancton III et IV s'opposent par leurs productions respectives : 150 à $250 \mathrm{~kg} /$ hectare pour Volvocales et Chlorococcales (CTR : $56 \%$ sur l'axe 2) contre 250 à $500 \mathrm{~kg} /$ hectare pour les Euglénophycées (CTR : $23 \%$ sur l'axe 2). Ces deux groupes sont en situation opposée aux plus fortes productions et aux Cyanobactéries (CTR : 26 \% sur l'axe 3). 


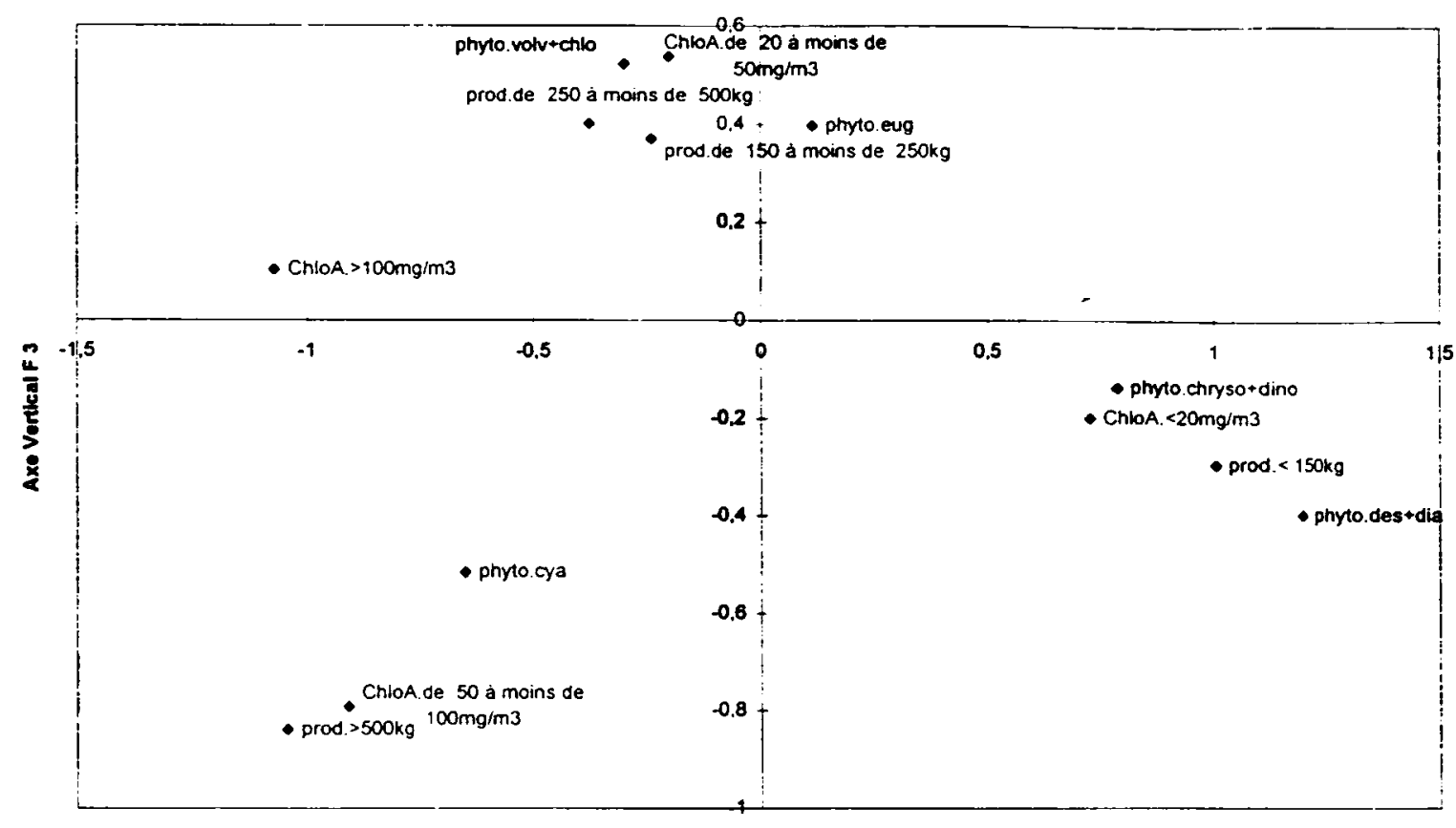

Axe Horizontal F 1

Figure 3

Relations entre les teneurs en chlorophylle a $\left(\mathrm{mg} / \mathrm{m}^{3}\right)$ et la production piscicole nette (kg/hectare) des étangs suivant les axes 1 et 3 .

\section{Figure 3}

Relations between chlorophyll $a\left(\mathrm{mg} / \mathrm{m}^{3}\right)$ concentration and fish net production in ponds (axes 1 and 3 of MCA).

Sur la base de ces informations, une grille de classification de la productivité potentielle des étangs peut être établie (Figure 4). Cette analyse précise et conforte les observations de terrain faites par BARBE et al. (1991) et SCHLUMBERGER (1993), qui proposent d'utiliser les groupes de phytoplancton en combinaison avec les teneurs en chlorophylle pour prévoir la production piscicole potentielle d'un étang de pisciculture.

La grille de classification proposée a été validée ensuite sur 8 étangs de pisciculture différents de ceux utilisés pour sa mise au point, situés dans d'autres zones géographiques, et présentant une large gamme de niveaux de productivité (Tableau II). Les niveaux de production estimés ont été établis également pour les étangs de la base de données d'origine.

Bien qu'une part de connaissance du terrain reste nécessaire, la gamme des niveaux de production prévus est relativement plus étroite que celle obtenue à partir d'autres critères (chlorophylle a, phosphore dissous). 


\section{Tableau II}

Application de la méthode proposée. Exemples sur 8 étangs. Seul le phosphore sous forme orthophosphate est dosé.

Remarques concernant les étangs notés * :

- EW5 : l'étang est entouré par une ceinture de macrophytes importante, la transparence au disque de Secchi est toujours élevée ;

- La Dame : la trop forte densité initiale de brochetons a décimé les gardons et les tanches, d'où une production globale inférieure à ce qui était escompté.

\section{Table II}

Application of the proposed method; examples on 8 ponds. Only orthophosphates are measured.

Remarks on ponds noted * :

- EW5 : This pond is surrounded by a thick belt of macrophytes, the Secchi disk visibility is always high ;

- La Dame : The initial density of pike fingerlings was too high, decimating the stock of roach and tench, consequently the final total production was lower than expected.

\begin{tabular}{|c|c|c|c|c|c|c|}
\hline SITES & $\begin{array}{l}\text { Surface } \\
\text { (ha) }\end{array}$ & $\begin{array}{l}\text { PO4 dissous } \\
(\mathrm{mg} / \mathrm{l})\end{array}$ & $\begin{array}{l}\text { Chl. } a \\
\left(\mathrm{mg} / \mathrm{m}^{3}\right)\end{array}$ & $\begin{array}{l}\text { Phytoplancton } \\
\text { (groupes dominants) }\end{array}$ & $\begin{array}{l}\text { Production } \\
\text { prévue }\end{array}$ & $\begin{array}{l}\text { efte ( } \mathrm{kg} / \mathrm{ha} \text { ) } \\
\text { effective }\end{array}$ \\
\hline \multicolumn{7}{|l|}{ LORRAINE } \\
\hline EW5 * & 11 & 0,03 & 110 & Chloroph., Cyanobactéries & $>500$ & 225 \\
\hline \multicolumn{7}{|l|}{ ALLIER } \\
\hline La Vilaine & 4,5 & 1 & 25 & Dinoph., Cyanobactéries & env. 250 & 265 \\
\hline Les Bruyères & 3,5 & 0,3 & 100 & Chlorophycées & 500 & 450 \\
\hline Les lles & 4,5 & 0,7 & 28 & Chloroph., Eugléno., Dinoph. & $<250$ & 220 \\
\hline \multicolumn{7}{|l|}{ DORDOGNE } \\
\hline Ménissoux & 0,6 & 0,2 & 11 & Chioroph., Cyanobactéries & env. 150 & 130 \\
\hline La Duche & 1,5 & 0,1 & 11 & Chloroph., Cyanobactéries & env. 150 & 150 \\
\hline La Dame * & 0,8 & 0.1 & 11 & Diatom., Chloroph., Dinoph. & $<100$ & 50 \\
\hline 4 Chemins & 0,7 & 0,03 & 115 & Chloroph., Dinophycées & env. 500 & 500 \\
\hline
\end{tabular}

\begin{tabular}{|c|c|c|c|c|c|}
\hline $\begin{array}{l}\text { Teneur en } \\
\text { chlorophylle-a } \\
\left(\mathrm{mg} / \mathrm{m}^{3}\right)\end{array}$ & $\begin{array}{l}\text { Groupe I : } \\
\text { diatomées et } \\
\text { desmidiées }\end{array}$ & $\begin{array}{l}\text { Ciroup II : } \\
\text { chrysophycees } \\
\text { et dimophycés }\end{array}$ & $\begin{array}{l}\text { Groupe III: } \\
\text { volvociales el } \\
\text { chlorococales }\end{array}$ & $\begin{array}{l}\text { Groupe IV: } \\
\text { cuglénophycés }\end{array}$ & $\begin{array}{l}\text { Groupe V: } \\
\text { cyanobactéries }\end{array}$ \\
\hline$<20 \mathrm{mg} / \mathrm{m}^{2}$ & & & & & \\
\hline 20$) .50 \mathrm{mg} / \mathrm{m}^{3}$ & & & & & \\
\hline $5(0) .1(x) \mathrm{mg} / \mathrm{m}$ & & & & & \\
\hline$>100 \mathrm{mg} / \mathrm{mi}^{\prime}$ & & & & & \\
\hline
\end{tabular}

Légende

\begin{tabular}{|c|}
\hline$<150 \mathrm{~kg} / \mathrm{ha}$ \\
\hline $150-250 \mathrm{~kg} / \mathrm{ha}$ \\
\hline $250-500 \mathrm{~kg} / \mathrm{ha}$ \\
\hline$>500 \mathrm{~kg} / \mathrm{ha}$ \\
\hline
\end{tabular}




\section{Figure 4}

Grille permettant de déterminer la production piscicole potentielle d'étangs (kg/ha/saison) à partir du groupe de phytoplancton dominant et de la concentration moyenne en chlorophylle a $\left(\mathrm{mg} / \mathrm{m}^{3}\right)$ pendant la saison de croissance.

\section{Figure 4}

Grid proposed to estimate the potential fish production ( $\mathrm{kg} / \mathrm{ha} / \mathrm{year}$ ) in a pond from the dominant phytoplankton group and the mean chlorophyll a concentration $\left(\mathrm{mg} / \mathrm{m}^{3}\right)$ during the growing season.

\section{MISE EN CEUVRE ET LIMITES DE LA MÉTHODE}

Les prélèvements peuvent se faire en un seul point de l'étang, depuis la digue, à la hauteur du dispositif de vidange, sans influence sur la qualité du résultat. La fréquence des campagnes de mesures peut être réduite, mais il est nécessaire que les échantillons soient collectés lors des maximums de prolifération du phytoplancton, lors du réchauffement de l'eau (avril-mai) et en fin d'été (août-septembre).

Si l'usage d'un spectrophotomètre est nécessaire pour la mesure de chlorophylle a après filtration et extraction à l'acétone (AFNOR, 1984), l'identification des groupes de phytoplancton ne pose pas de difficultés particulières, puisqu'on évite dans une large mesure les difficultés de la taxonomie.

La méthode présentée ici permet, à partir du phytoplancton (groupe prédominant et concentration en chlorophylle a dans le milieu) de définir une gamme de valeurs pour la production piscicole nette potentielle d'un site donné. Rappelons qu'elle a été établie à partir d'étangs avec polyculture classique : $C$. carpio, $R$. rutilus ou erythrophtalmus, $T$. tinca, avec généralement $E$. lucius comme carnivore, qui constitue 5 à $10 \%$ de la biomasse. Une fertilisation organique et/ou minérale plus ou moins intense est pratiquée, mais il n'y a pas de nourrissage direct du poisson.

Le stock de poisson introduit, en quantité (et qualité), espèces, classes d'âge et proportions relatives, doit tenir compte des potentialités de l'étang, et a un rôle important dans la conformité du résultat pratique avec la prévision faite. L'expérience montre qu'en absence de carnivore, la production des Cyprinidés augmente de 20 à $30 \%$ dans la mesure où les ressources alimentaires (zooplancton, macroinvertébrés) ne sont pas limitantes (SCHLUMBERGER et al., 1999). Il est évident que des mortalités anormales se répercuteront sur le résultat final.

Les évaluations de production sur un étang seront d'autant plus sous-estimées que le développement des macrophytes y est notable (plus de $10 \%$ de la surface en eau), cas par exemple de l'étang EW5 (Tableau II). En effet, sur les étangs riches en macrophytes, les apports d'azote et de phosphore bénéficient essentiellement aux végétaux supérieurs, restreignant le développement du phytoplancton ; un rapport $\mathrm{C} / \mathrm{N}$ dissous élevé, grâce aux débris végétaux, favorise le développement bactérien, à partir duquel se développe un peuplement peu dense de zooplancton. Sur de tels plans d'eau, ce sont les macroinvertébrés du benthos et surtout ceux présents dans la végétation submergée (organismes phytophiles), dont la productivité est plus faible que celle du zooplancton, qui assurent l'essentiel des ressources alimentaires des poissons. La chaîne trophique " classique " basée sur le phytoplancton et aboutissant au poisson prend alors une importance relative d'autant plus limitée que la part de la surface de l'étang occupée par les macrophytes aquatiques est forte. Cette méthode d'estimation de la production 
piscicole n'est applicable que sur des étangs où sont élevées des espèces européennes de Cyprinidés à des densités compatibles avec les ressources trophiques du milieu. Le savoir-faire du gestionnaire de l'étang joue un rôle important. Un stock de poissons trop élevé peut induire par effet "top-down" la disparition de grandes formes du zooplancton (Cladocères filtreurs à large spectre de proies) qui se répercutera sur le peuplement algal (augmentation de la biomassae algale et de la turbidité). Inversement, une réduction du stock de poissons zooplanctonophages permet aux Cladoceres de grande taille d'exercer une forte pression de broutage sur le phytoplancton de petite dimension, aboutissant à une plus grande transparence de l'eau.

Dans le cas d'élevage de poissons filtreurs détritivores (par exemple Tilapias en régions tropicales) des informations intéressantes pourraient être obtenues en appliquant aux teneurs en MES dans les étangs une méthode similaire à celle proposée ici.

\section{CONCLUSION}

Les diverses méthodes existantes d'estimation de la production piscicole en plan d'eau ne sont pas applicables aux étangs de pisciculture, milieux fortement anthropisés. L'emploi de données quantitatives (concentration en chlorophylle a) et qualitatives (groupes d'algues principaux présents) à partir du phytoplancton permet par contre une prévision de cette production avec une précision suffisante pour le gestionnaire sur une large gamme d'étangs (dimensions, productivité). Cela confirme le rôle des groupes algaux en qualité et densité comme indicateurs du niveau trophique de milieux aquatiques aussi anthropisés que les étangs de pisciculture à production cyprinicole.

\section{BIBLIOGRAPHIE}

AFNOR, 1984. Dosage de la chlorophylle a et des phéopigments par spectrométrie d'absorption moléculaire. Norme T90-117. $7 \mathrm{p}$.

AVNIMELECH Y., KOCHVA M., DIAG S., 1994. Development of controlled intensive aquaculture systems with a limited water exchange and adjusted carbon to nitrogen ratio. Bamidgeh, 46 (3), 119-131.

BALVAY G., 1995. Ressources et comportements alimentaires des poissons. In : Limnologie générale, POURRIOT R. et MEYBECK M. (Eds), Masson, Paris, 588-607.

BARBE J., LAVERGNE E., ROFES G., LASCOMBE M., RIVAS J., BORNARD C., De BENEDETIS J., 1990. Diagnose rapide des plans d'eau. Informations techniques du CEMAGREF. $N^{\circ} 79$, note 2, 1-8.

BARBE J., CAMUS J.C., SCHLUMBERGER O., 1991. Facteurs de production piscicole en étang et gestion. Echo-système, 20, 3-25.

BERARD A., 1993. Effets d'une fertilisation riche en matières organiques azotées sur les relations trophiques (bactéries, phytoplancton, zooplancton) dans un étang de pisciculture. Thèse Mus. Nat. Hist. Nat., Paris. 215 p.

BILLARD R., MARIE D., 1980. La qualité des eaux de l'étang de pisciculture et son contrôle. In : La pisciculture en étang, BILLARD R. (ed.), INRA Publ., Paris, 107-127.

BIRO P., VOROS L., 1990. Trophic relationships between primary producers and fishyields in lake Balaton. Hydrobiologia, 191, 213-221.

BOYD C.E., 1982. Water quality management for pondfish culture. Elsevier Scientific Publishing Company, $318 \mathrm{p}$. 
C.A.R.A., CEMAGREF., 1992. Programme expérimental et de démonstration de pisciculture d'étang dans la Double (Dordogne). Rapport 1988-1991. Compagnie d'Aménagement Rural d'Aquitaine, Bordeaux, CEMAGREF, division Aquaculture et Pêche, Montpellier, $70 \mathrm{p}$.

CEMAGREF., 1986. Développement des productions aquacoles en Petite Woëvre. CEMAGREF, division Qualité des Eaux, Lyon, $140 \mathrm{p}$.

CEMAGREF., 1988a. Etude de caractérisation des étangs de pisciculture du Forez : analyse des facteurs de production ; application à la gestion piscicole des étangs. Campagne 1987. CEMAGREF, division Qualité des Eaux, Lyon, 123 p.

CEMAGREF., 1988b. Rôle des facteurs du milieu sur l'état sanitaire et la croissance des jeunes carpes en étang (Jointe et Devant). CEMAGREF, division Aquaculture et Pêche, Lyon, $52 \mathrm{p}$.

CEMAGREF., 1989. Rôle des facteurs du milieu sur l'état sanitaire et la croissance des poissons élevés en étang. CEMAGREF, division Aquaculture et Pêche, Lyon, $46 \mathrm{p}$.

CEMAGREF., 1990a. Développement des productions aquacoles en Petite Woëvre. Etude de milieu 1985-1987. CEMAGREF, division Aquaculture et Pêche, Lyon, 62 p.

CEMAGREF., 1990b. Etude de la caractérisation des étangs de pisciculture de la Bresse jurassienne. CEMAGREF, division Aquaculture et Pêche, Lyon, 95 p. + annexes.

CEMAGREF., 1991a. Etude de caractérisation des étangs de pisciculture du Forez. Campagne 1989. CEMAGREF, division Biologie des Ecosystèmes Aquatiques, Lyon, $59 \mathrm{p}$.

CEMAGREF., 1991b. Etude des possibilités de production des étangs du Bourbonnais (Allier). Rapport final. SICA Piscicole de l'Allier ; DDAF de l'Allier ; CEMAGREF, division Aquaculture et Pêche, Montpellier, $45 \mathrm{p}$. + annexes.

DOWNING J.A., PLANTE C., LALONDE S., 1990. Fishproduction correlated with primary productivity, not the morphoedaphic index. Can. J. Fish. Aquat. Sci., 47, 1929-1936.

GRYGIEREK E., 1979. Plankton as an ecological indicator of the influence of farming measures on pond biocenosis. Pol. Ecol Stud., 5 (4), 77-140.

HANSON J.M., LEGGETT W.C., 1982. Empirical prediction of fish biomass and yield. Can. J. Fish. Aquat. Sci., 39, 257-263.

HUET M., 1949. Appréciation de la valeur piscicole des eaux douces. Trav. Stat. Rech. Eaux et Forêts, Groenendaal. Série D, 10,55 p. 41 fig.

HUET M., 1964. The evaluation of the fish productivity in freshwater (the coefficient of productivity k). Verh. Internat. Verein. Limnol., 15, 524-528.

HUET M., 1970. Traité de pisciculture. De WYNGAERT (ed.), Bruxelles, 718 p.

JONES J.R., HOYER M.V., 1982. Sportfish harvest predicted by summer chlorophyll a concentration in Midwestern lakes and reservoirs. Trans. Amer. Fish. Soc., 111, 176-179.

LAZZARO X., LACROIX G., 1995. Impact des poissons sur les communautés aquatiques. In : Limnologie générale, POURRIOT R. et MEYBECK M. (Eds), Masson, Paris, 648-686.

LEACH J.H., DICKIE L.M., SHUTER B.J., BORMAN V., HYMAN J., LYSACK W., 1987. A review of methods for prediction of potential fish production with application to the Great lakes and lake Winnipeg. Can. J. Fish. Aquat. Sci., 44 (suppl. 2), 471-485.

MATUSZEK J.E., 1978. Empirical predictions of fish yields of large North American lakes. Trans. Amer. Fish. Soc., 107, 385-394.

MOREAU J., DE SILVA S., 1991. Predictive fish yield models for lakes and reservoirs of the Philippines, Sri Lanka and Thailand. FAO Fish. Tech. Paper 319. FAO Rome, $42 \mathrm{p}$. 
NORTHCOTE T.H., LARKIN P.A., 1956. Indices of productivity in British Columbia lakes. J. Fish. Res. Bd. Can., 13, 515-540.

OCDE, 1982. Eutrophisation des eaux. Méthodes de surveillance, d'évaluation et de lutte contre l'eutrophisation. OCDE, Paris, $164 \mathrm{p}$.

OGLESBY R.T., 1977a. Phytoplankton standing crop and annual productivity as functions of phosphorus loading and various physical factors. J. Fish. Res. Bd. Can., 34, 2255-2270.

OGLESBY R.T., 1977b. Relationships of fish yields to lake phytoplankton standing crop, production, and morphoedaphic factors. J. Fish. Res. Bd. Can., 34, 2271-2279.

OGLESBY R.T., 1982. The morphoedaphic index - concept and practices. Trans. Amer. Fish. Soc., 111 (special section), 133-175.

RAWSON D.S., 1951. The total mineral content of lake waters. Ecology, 32, 669-672.

RAWSON D.S., 1952. Mean depth and the fish production of large lakes. Ecology, 33 (4), 513-521.

RYDER R.A., KERR S.R., LOFTUS K.H., REGIER H.A., 1974. The morphoedaphic index, a fish yield estimator - review and evaluation. J. Fish. Res. Bd. Can., 31, 663-688.

SCHLUMBERGER O., 1993. Mémento de pisciculture d'étang. CEMAGREF, Série “Etudes " Ressources en eau n7. CEMAGREF-DICOVA, Antony. $166 \mathrm{p}$.

SCHLUMBERGER O., RIGAUD C., MASSE J., 1999. Valorisation des étangs de pisciculture en Dordogne. Bilan des expérimentations 1995-97. CEMAGREF/AMVEP-SICA Aquacole d'Aquitaine. Montpellier. 75 p. + annexes.

WURTZ A., 1958. Peut-on concevoir la typification des étangs selon les mêmes bases que celle des lacs? Verh. Internat. Ver. Limnol., 13, 381-393.

WURTZ-ARLET J., 1980. La fertilisation des étangs. In : La pisciculture en étang, BILLARD R. (ed.), INRA Publ., Paris, 99-106. 